\title{
BEAM POSITION MEASUREMENTS AT LOS ALAMOS: ISOTOPE PRODUCTION FACILITY AND SWITCHYARD KICKER UPGRADE*
}

\author{
J. D. Gilpatrick, D. Barr, D. Martinez, J. F. O’Hara, R. B. Shurter, M. Stettler, LANL, Los Alamos, \\ New Mexico, USA.
}

\section{Abstract}

The Los Alamos Neutron Science Center (LANSCE) is installing two beam lines to both improve operational tuning and provide new capabilities. The Isotope Production Facility (IPF) will provide isotopes for medical purposes by using the $\mathrm{H}^{+}$beam spur at $100 \mathrm{MeV}$ and the Switchyard Kicker Upgrade (SYK) will allow the LANSCE $800-\mathrm{MeV} \mathrm{H} \mathrm{H}^{-}$beam to be rapidly switched between various beam lines within the facility. The beam position instrumentation for both of these beam lines uses a micro-stripline beam position monitor (BPM) with a 50 $\mathrm{mm}$ or $75-\mathrm{mm}$ radius. The cable plant is unique in that it unambiguously verifies the operation of the complete position instrumentation. The processing electronics use a $\log$ ratio technique with error correction such that it has a dynamic range of $-12 \mathrm{dBm}$ to $-85 \mathrm{dBm}$ with errors less than $0.15 \mathrm{~dB}$ within this range. This paper will describe the primary components of these measurement systems and provide initial data of their operation.

\section{IPF AND SYK BEAM LINES}

The LANSCE facility is constructing an IPF to provide radioisotopes for diagnosis and treatment of diseases [1]. This spur beam line starts at the $100-\mathrm{MeV}$ transition region of the accelerator and transports $\mathrm{H}$ beam to a target area where samples may be irradiated and safely handled. The new beam line contains eight BPMs used to diagnose the beam's position throughout the transport and verify the beam's placement on the target/sample region during either a $10-\mathrm{kHz}$ raster or static operation.

At the end of the $800-\mathrm{MeV}$ LANSCE accelerator, a switchyard directs the beam to either Line D or to Line $\mathrm{X}$ [2]. Line D contains such facilities as the Lujan Neutron Scattering Center and Line X contains such facilities as the Proton Radiography and Ultra Cold Neutron Projects. Prior to the installation of the upgraded switchyard kicker beam line, a lengthy process was required to reconfigure the switchyard beam line in order to transport the beam to either Lines D or X. With the completion of the SYK, LANSCE can now simultaneous provide $\mathrm{H}^{-}$beams to both beam lines by rapidly switching between them using fast kickers located in the new SYK. Three additional BPMs, located in the new SYK, provide beam position information to the facility operators.

Table 1 summarizes key operational requirements of the position measurements for IPF and SYK (a more complete table is in reference [3]). While for IPF and SYK, the beam species are of opposite signs, many

\footnotetext{
* Work supported by the U.S. DoE, Defense Programs and the U.S.

DoE, Energy Research.
}

measurement parameters are either the same or very similar, e.g. bunching frequency of $201.25 \mathrm{MHz}$ and data acquisition rate of $<10 \mathrm{~Hz}$. However, note the IPF $\mathrm{H}^{\dagger}$ beam does not have any chopped structure in it, resulting in lower IPF bandwidths requirements for measuring rastered beams. Also note the dynamic range of the both types of signals is larger than the macropulse beam current range would indicate. This range difference is due to the amount of additional range required to cover off centered beam conditions at either the highest or lowest beam currents. The SYK chopping structure for the various experimental beam lines can be quite complex. If allowed, these chopped-beam structures could further broaden the SYK BPM dynamic range requirements. However, it was decided that monitoring the position of these chopped beams was unnecessary as long as there was a non-chopped tuning beam available.

Table 1: Overall position measurement requirements.

\begin{tabular}{|c|c|c|}
\hline Parameter & IPF & SYK \\
\hline Macropulse Length (ms) & 0.05 to 1 & 0.15 to 1.2 \\
\hline Chopped Beam Rate (MHz) & N/A & $2.8 \& 5.6$ \\
\hline Chopped Beam DF (\%) & N/A & 22 to 56 \\
\hline $\begin{array}{c}\text { Macropulse Pulse Beam } \\
\text { Current (mA) }\end{array}$ & 16.5 to 0.1 & 13 to 0.1 \\
\hline Base Bandwidth (kHz) & 15 & $\sim 2500$ \\
\hline $\begin{array}{c}\text { Position Precision/Accuracy } \\
(\% \text { of pipe radius) }\end{array}$ & $0.3 / \sim 3$ & $0.25 / \sim 2$ \\
\hline $\begin{array}{c}\text { Beam Pipe Radius (mm) } \\
\text { Position Measurement } \\
\text { Dynamic Range (dB) }\end{array}$ & $50.4,76.2$ & 50.4 \\
\hline
\end{tabular}

\section{BPM BEAMLINE COMPONENTS}

A previous paper details the BPM's mechanical construction and mapping of the IPF BPMs, this paper only briefly discusses the SYK BPM's characteristics [3]. Fig. 1 and 2 show a SYK BPM and its associated beam line components in picture and schematic form. The SYK BPMs were characterized to have a $0.627 \mathrm{~dB}$ per $\mathrm{mm}$ sensitivity and typical offsets < +/- $0.1 \mathrm{~mm}$. The sensitivity is within $5 \%$ of the theoretical $0.661 \mathrm{~dB}$ per $\mathrm{mm}$. Since the SYK and IPF BPMs have feed-throughs at both the downstream and upstream end of each of the four electrodes, a unique method of measurement operation is performed to monitor the BPM's condition during beam operation. As shown in Fig. 2, there is a completely 
unambiguous signal path for power measurements to and from the processor module through each BPM electrode.

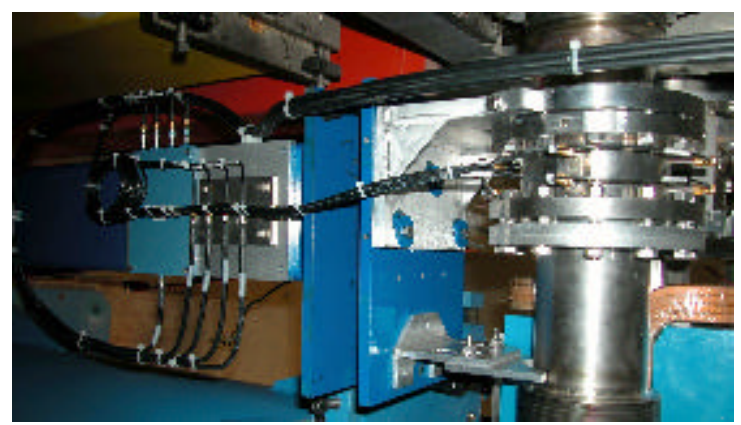

Figure 1. A SYK BPM (oriented vertically in figure) is shown with its associated verification hardware.

The 20-dB attenuators in each electrode signal path provide both additional RF divider leg isolation and $50-\Omega$ termination for the BPM electrode downstream ports. The typical round trip attenuation is $36 \mathrm{~dB}+/-1 \mathrm{~dB}$ and with an injection signal power of $-25 \mathrm{dBm}$, the resulting verification power measurements are performed at -61 $\mathrm{dBm}$. Since the components in this loop are linear, a single mid-dynamic-range power measurement for each cable/electrode loop path is sufficient to determine the health of the any of the serial components within the loop. If a cable is inadvertently crimped or a BPM electrode is injured to the point of losing its 50 -ohm characteristics, the total loop attenuation will change. With the help of a software process, this attenuation measurement is performed on an hourly basis in-between beam pulses so that facility operators always have a quantitative method of detecting BPM and cable health.

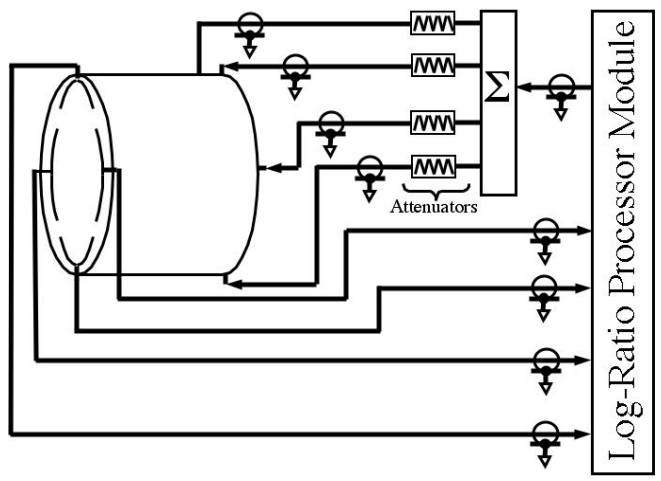

Figure 2. This simple block diagram shows the verification test hardware.

\section{LOG-RATIO ELECTRONIC PROCESSOR}

The log-ratio electronics used in this VXI-crate-based processor module has a digital motherboard with on-board digital signal processor (DSP) daughter cards, widebandwidth analog-front-end (AFE) circuitry that uses a logarithmic amplifier in each of the four channels, and an on-board 201.25-MHz oscillator on a calibrator daughter card. Fig. 3 shows a simplified schematic of the AFE and calibrator daughter cards. Since all of the components in the calibrator circuitry are solid-state devices, the multi- step calibration process can be accomplished in the $8.3 \mathrm{~ms}$ between beam pulses.

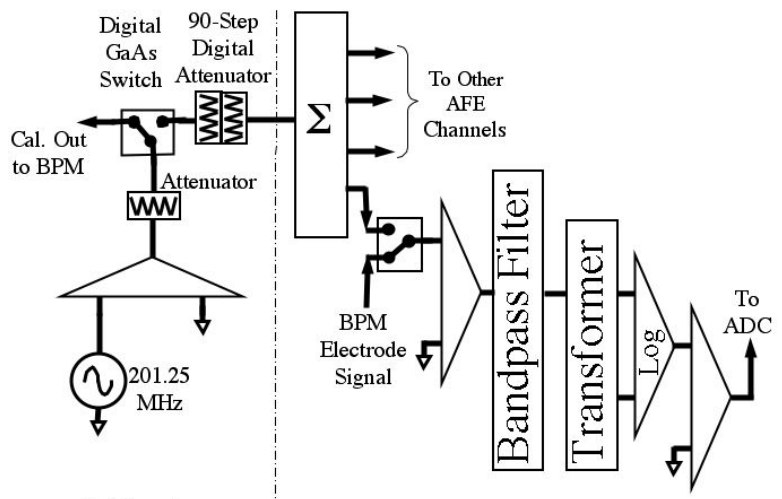

Calibrator

Daughter Card

AFE Daughter Card: Single

Channel

Figure 3. Simplified functional block diagram of the AFE and calibrator daughter cards.

Fig. 4 shows the result of the calibration circuit changes. The deterministic and systematic calibrator errors are subtracted from each of the AFE channels during the software calibration procedure. The error bars, typically $<0.1 \mathrm{~dB}$, represent the random errors and uncertainty of the calibrator output signal power.

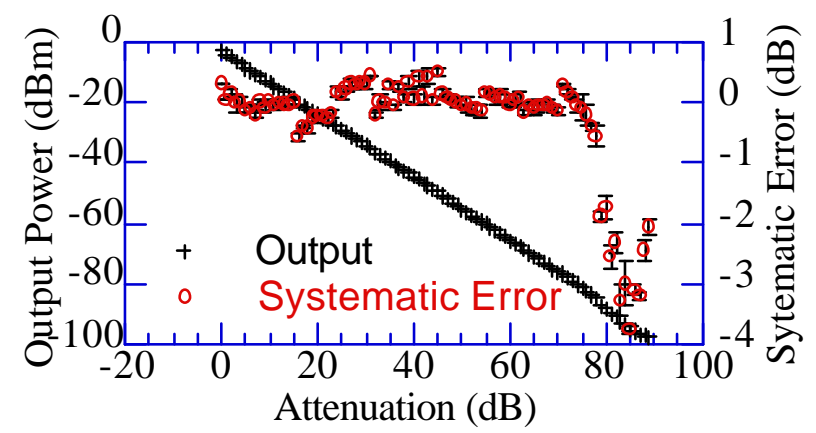

Figure 4. Displayed is a calibrator output signal power and its systematic error as a function of dynamic range.

The present AFE circuitry measures very low-power signals with a wide bandwidth. A Temex $201.25-\mathrm{MHz}$ band-pass filter was placed between the input transformer to the Analog Devices AD8307 log amplifiers and a GALI-52 Mini-Circuits pre-amplifier. Since the 201 $\mathrm{MHz}$ harmonic-signal power was lower than initially expected, the band-pass filter was placed to reduce the broadband digital noise finding its way into the log-amp input and not to limit higher harmonic signal power to the preamplifier. These 4.5-MHz-bandwidth filters also have short rise and fall times, typically $76-$ and 132-ns respectively, providing sufficient bandwidth to measure various chopped-beam conditions in the SYK beam line.

Fig. 5 shows the result of two subtracted module channels, resulting in the log-ratio process, prior to a 90 $\mathrm{dB}$ software calibration using the circuitry shown in Fig. 3. The data labeled "Pre-Cal Error" are the residual deterministic errors from a pure logarithmic function. 
These errors are primarily due to the log-amp's logarithmic non-conformity and minor thermal variations and are subtracted out during the calibration routine. The Pre-Cal errors are shown for a centered-beam and an offcentered beam condition. The data are plotted as a function of input signal power, in $\mathrm{dBm}$, where a $1 \mathrm{~mA}$ current will occur at approximately $-55 \mathrm{dBm}$. Also plotted in Fig. 5 are the random error data, the ultimate limitation to the calibration process and measurement precision. Note that an axis is calibrated to within a $<0.15$ $\mathrm{dB}$ from approximately -12 to $-85 \mathrm{dBm}$. In terms of positional error and beam current applied to the $50-\mathrm{mm}$ IPF and SYK BPMs, this is equivalent to a $<0.25-\mathrm{mm}-\mathrm{rms}$ error over a $\sim 20$ to $0.1 \mathrm{~mA}$ current range.

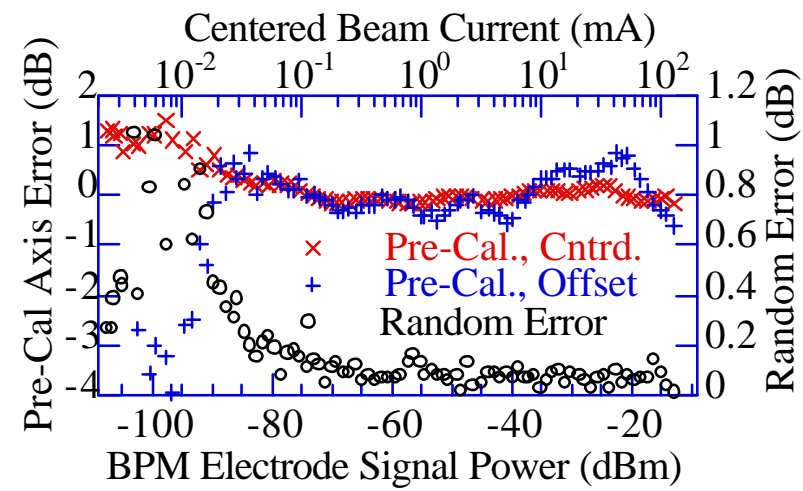

Figure 5. This graph shows the systematic errors prior to and the random errors after a software calibration.

\section{POSITION MEASUREMENT SOFTWARE}

The calibration procedure is controlled with a personal computer input/output controller (PCIOC). This procedure uses the same calibrator daughter-card signalsource shown in Fig. 3 that the verification measurement uses but in this case, digital-controlled step attenuators are used to step through a $90-\mathrm{dB}$ range. This procedure was first described in a previous paper [5].

A National Instruments LabVIEW virtual instrument (VI) software process continuously runs on the PCIOC that performs the calibration and verification procedures, repeating on an approximate hourly basis. Upon receiving a timing signal through each VXI processor module, the VI acquires the digital information from the four channels' analog-to-digital converters (ADC) via the digital signal processor (DSP) daughter cards after it has "armed" the DSPs to perform the acquisition [5]. These data are then calibrated by addressing a specific memory location in a 4096 location RAM look-up table (RAMLUT) that contains the correct calibrated data (in counts) on the digital signal processor (DSP) daughter cards. These RAMLUTs remove the systematic "Pre-Cal Errors" for each of the four-processor channels. After the calibration has been completed on all four processor channels, opposite-electrode calibrated-signal powers are then digitally -subtracted to produce a calibrated log-ratio signal for a single axis. Since both IPF and SYK are pulsed beam facilities, a separate timing signal between beam pulses initiates the calibration and verification sub-
VI. This calibration process loads a RAMLUT while another previously loaded RAMLUT is used to provide calibrated position information. After the calibration RAMLUTs have been filled, loaded, and applied to incoming data, another sub-VI switches the appropriate GaAs RF switches in the AFE and Calibrator daughter cards so that the verification procedure checks the health of the cables and BPM electrodes as described earlier.

Finally, the VI serves the data via a portable channel access sub-VI written to interface with the accelerator control system, EPICS (Experimental \& Physics Industrial Control System) [6]. The VI, first implemented for the low energy demonstration accelerator at Los Alamos, also allows the facility operators to initiate an "on-demand" calibration procedure and verification test, however, for the "on-demand" verification test to be accurate, the beam through the BPM is turned off.

\section{SUMMARY}

This paper describes a beam position instrumentation presently installed in the LANSCE IPF and SYK beam lines. The measurement system uses 50- and 75-mm radius micro-stripline BPMs, an unambiguous verification process that monitors the measurement system's beamline-hardware health, and an automatic calibration process that removes deterministic and thermal errors on a periodic basis without operator intervention. It has a dynamic range of $>73 \mathrm{~dB}$ as defined by errors that are $<0.15 \mathrm{~dB}$ (or $<0.25 \mathrm{~mm}$ ). Even with this wide dynamic range, the instrumentation bandwidth has been measured to be $>2.5 \mathrm{MHz}$.

\section{REFERENCES}

[1] R.C. Heaton, E.J. Peterson, "Construction of a New Isotope Production Facility,” pp. 112 - 115, LANSCE Activity Report 2001, LA-13943-PR, December, 2001.

[2] D.H. Fitzgerald, M.S. Gulley, "LANSCE Switchyard Kicker Project," pp. 124 - 125, LANSCE Activity Report 2001, LA - 13943-PR, December, 2001.

[3] J. F. O'Hara, et al., “The Mechanical Design and Preliminary Testing Results of Beam Position Monitors for the LANSCE Isotope Production Facility and Switchyard Kicker Projects.," BIW2002, Upton, NY, May, 2002.

[4] R. Shurter, et al., "Analog Front-End Electronics For Beam Position Measurement On The Beam Halo Measurement," PAC2001, pp.1369-1371, Chicago, IL, June, 2001.

[5] D. Barr, et al., "Upgrade To Initial BPM Electronics Module And Beamline Components For Calibration Of The LEDA Beam Position Measurements," PAC2001, pp.1369-1371, Chicago, IL, June, 2001.

[6] K.U. Kasemir, et al., "Integrating LabVIEW into a Distributed Computing Environment," ICALEPCS 2001, San Jose, CA. LA-UR-01-5905. 